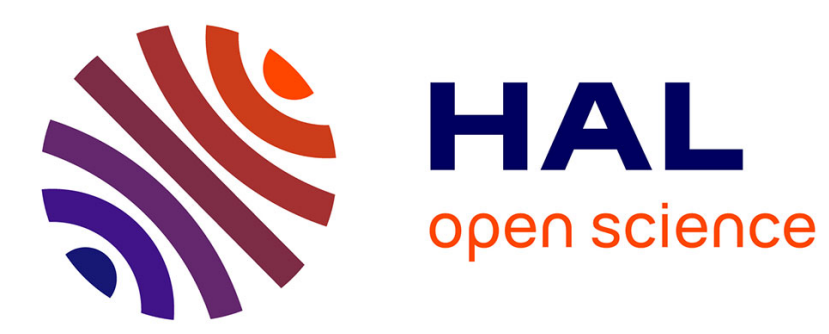

\title{
L'effet des transferts des migrants sur la déforestation dans les pays en développement
}

\author{
Laetitia Duval, François-Charles Wolff
}

\section{To cite this version:}

Laetitia Duval, François-Charles Wolff. L'effet des transferts des migrants sur la déforestation dans les pays en développement. Revue d'Economie du Développement, 2009, 17 (3), pp.109. 10.3917/edd.233.0109 . hal-03185426

\section{HAL Id: hal-03185426 \\ https://hal.science/hal-03185426}

Submitted on 30 Mar 2021

HAL is a multi-disciplinary open access archive for the deposit and dissemination of scientific research documents, whether they are published or not. The documents may come from teaching and research institutions in France or abroad, or from public or private research centers.
L'archive ouverte pluridisciplinaire HAL, est destinée au dépôt et à la diffusion de documents scientifiques de niveau recherche, publiés ou non, émanant des établissements d'enseignement et de recherche français ou étrangers, des laboratoires publics ou privés. 


\title{
L'effet des transferts des migrants sur la déforestation dans les pays en développement
}

\author{
Impact of Remittances on Deforestation \\ in Developing Countries
}

\author{
Laetitia Duval ${ }^{*}$ \\ LEMNA, Université de Nantes \\ François-Charles Wolff ** \\ LEMNA, Université de Nantes ; CNAV et INED, Paris
}

Cet article s'intéresse à l'effet des transferts monétaires des migrants internationaux sur l'environnement, à travers l'exemple de la déforestation dans les pays en développement. D'un point de vue théorique, l'effet des transferts migratoires sur la réduction des surfaces forestières apparaît indéterminé. Nous examinons cette relation à partir d'un échantillon comprenant 102 pays pour la période allant de 1990 à 2005. Le profil observé pour le PIB par tête s'avère contraire à l'hypothèse de courbe environnementale de Kuznets, tandis que la part des transferts migratoires reçus dans le PIB vient réduire le taux de déforestation pour l'ensemble des pays considérés.

Mots clés : déforestation, transferts migratoires, pays en développement.

Classification JEL: O13, O15, Q23

* Correspondance LEMNA, Université de Nantes, BP 52231 Chemin de la Censive du Tertre, 44322 Nantes Cedex. E-mail: duval.laetitia@yahoo.com

** LEMNA, Université de Nantes; CNAV et INED, Paris. E-mail: francois.wolff@univnantes.fr http://www.sc-eco.univ-nantes.fr/ fcwolff

Nous tenons à remercier deux rapporteurs anonymes pour leurs nombreuses remarques et suggestions qui nous ont été des plus utiles pour les révisions de ce texte. Nous remercions également Jean-Louis Combes, José de Sousa, Frédéric Docquier, El Mouhoub Mouhoud, Claudia Senik et Fabien Tripier pour leurs différents commentaires et remarques, ainsi que les participants à l'atelier international « Déforestation tropicale : déterminants et implications politiques » qui s'est tenu le 14 novembre 2008 au CERDI à Clermont-Ferrand. 
In this paper, we study the impact of remittances on environment, with a focus of deforestation in developing countries. From a theoretical point of view, the role of remittances on deforestation is a priori unclear. Then, we investigate this relationship using a sample of 102 developing countries covering the period from 1990 to 2005. The profile observed for the GDP per capita does not support the hypothesis of the environmental Kuznets curve, while the share of remittances in GDP reduces the rate of deforestation.

Key words: deforestation, remittances, developing countries.

\section{INTRODUCTION}

Le montant des transferts monétaires effectués par les migrants internationaux à destination des pays en développement, encore appelés remittances, n'a cessé d'augmenter ces dernières années. Selon la Banque Mondiale (2008), il a été multiplié par six entre 1990 et 2005, passant de 31,1 à 194,7 milliards de dollars courants. Pour avoir un ordre de grandeur, les transferts migratoires représentent pour les pays en développement une source de financement extérieur deux fois supérieure à l'aide au développement qu'ils reçoivent et équivalente à près des deux tiers de leurs investissements directs étrangers entrants (Ratha, 2005). Si de tels flux financiers devraient avoir des retombées économiques positives sur les pays d'origine des migrants internationaux, les études sur le sujet sont en réalité contrastées car leurs effets sont multiples et parfois contradictoires (Rapoport et Docquier, 2006).

La question de l'impact des transferts migratoires sur les piliers du développement que constituent l'éducation, la santé, la culture ou l'environnement reste largement ouverte à ce jour (Gubert, 2005). Si Beine et alii (2008) montrent que les transferts migratoires augmentent le rendement attribué à l'éducation, Faini (2007) note qu'ils diminuent avec la proportion de migrants qualifiés. En revanche, très rares sont les études relatives aux effets potentiels de ces transferts migratoires sur l'environnement dans les pays en développement.

Pourtant, ces liens existent a priori. Le rapport de Stern (2007) relatif aux répercussions du réchauffement climatique indique que près de 200 millions de personnes pourraient migrer d'ici $2050^{1}$. Curieusement, la prise en compte des flux financiers que les migrants internationaux versent à leur pays d'origine est totalement passée sous silence. Pourtant, les populations les plus

Le rapport de Stern (2007) a mis en avant le concept de réfugiés environnementaux. Cette catégorie de migrants internationaux apparait toutefois dès les années 1980 dans un rapport publié par le Programme des Nations Unies pour l'Environnement (El-Hinnawi, 1985). Elle fait néanmoins l'objet de débats car aucun statut légal ne leur est à ce jour attribué. En effet, selon la Convention de Genève (1951), un réfugié est défini comme 
nombreuses qui sont concernées par les changements environnementaux sont celles qui restent sur place, et à ce titre, l'argent de la migration internationale peut avoir des répercussions significatives sur l'environnement local.

Dans cet article, nous étudions le rôle des transferts migratoires sur l'environnement, à travers l'exemple de la déforestation dans les pays en développement. Plusieurs raisons expliquent ce choix. Tout d'abord, la part des transferts migratoires à destination des pays en développement est en croissance régulière, de 45,2 \% en 1990 à 75,7 \% en 2007 (Banque Mondiale, 2008). Ensuite, les pays en développement sont ceux qui sont les plus vulnérables aux dégradations environnementales: si les pays du monde ont perdu $3,07 \%$ de leur superficie forestière sur la période 1990-2005 (FAO, 2005), les données masquent des différences importantes entre pays en développement et pays développés. L'Afrique a perdu $9,1 \%$ de sa superficie forestière sur cette période, tandis que l'Europe a augmenté la sienne de 1,2\%.

Enfin, les forêts jouent un rôle essentiel dans la subsistance des populations rurales et pauvres des pays en développement car elles sont aussi une source d'alimentation (viandes, légumes, fruits, etc.). La Banque Mondiale estime à plus d'un milliard le nombre de personnes qui vivent dans les forêts ou à proximité (World Bank, 2006). En outre, la Banque Mondiale estime à plus de 2 milliards le nombre de personnes qui dépendent du bois pour leurs besoins énergétiques, principalement pour cuisiner et se chauffer (World Bank, 2006).

La relation entre transferts migratoires et environnement s'avère indéterminée a priori. En contribuant à la croissance économique des pays en développement, les flux financiers de la migration internationale peuvent accélérer l'industrialisation et le développement urbain, portant ainsi atteinte aux superficies forestières. À l'inverse, dans la mesure où ils atténuent la pauvreté des populations qui en bénéficient (Adams et Page, 2005), les flux financiers de la migration internationale peuvent réduire la pression sur l'utilisation des terres et ainsi freiner la déforestation dans les pays en développement.

Pour étudier cette relation entre transferts migratoires et déforestation, nous menons une étude empirique auprès d'un échantillon de 102 pays en développement couvrant la période allant de 1990 à 2005, à partir de données fournies par la Food and Agriculture Organization (FAO). Les données de transferts migratoires proviennent de la Banque Mondiale (2008) et correspondent à la part des transferts migratoires reçus par chaque pays en pourcentage du PIB.

\footnotetext{
« toute personne craignant avec raison d'être persécutée du fait de sa race, de sa religion, de sa nationalité, de son appartenance à un certain groupe social ou de ses opinions politiques " (Article 1, section A, paragraphe 2). Pour de plus amples développements, se reporter à Black (2001).
} 
La déforestation a fait l'objet d'une littérature substantielle. Le sujet a investi le champ de la discipline économique dès le milieu des années 1980, autour de deux axes. Le premier s'intéressait aux relations qu'entretiennent la croissance économique et l'environnement à travers les courbes environnementales de Kuznets, tandis que le second portait sur les explications apportées à la déforestation. Si ces questions ont été largement étudiées d'un point de vue empirique (Angelsen, 1999 ; Bhattarai et Hammig, 2001 ; Azomahou et Nguyen Van, 2007), aucun consensus n'émerge à ce jour. Au regard de la littérature existante, notre travail est le premier à s'interroger sur le rôle des transferts migratoires sur l'environnement, à travers la déforestation.

Les résultats de notre étude économétrique indiquent que l'effet observé pour le PIB par tête s'avère contraire à l'hypothèse de courbe environnementale de Kuznets et que la part des transferts migratoires reçus dans le PIB vient réduire le taux de déforestation pour l'ensemble des pays considérés. Le reste de cet article est organisé de la façon suivante. Dans la section 1, nous présentons une revue de la littérature relative aux déterminants de la déforestation, en incluant le rôle potentiel des transferts migratoires. Dans la section 2 , nous décrivons les données utilisées et le modèle économétrique retenu. Les résultats des estimations sont discutés dans la section 3. Enfin, nous concluons dans la section 4 .

\section{LES DÉTERMINANTS DE LA DÉFORESTATION}

La revue de la littérature sur les déterminants de la déforestation révèle qu'il n'existe pas de consensus permettant de savoir quelles variables inclure dans un modèle empirique ${ }^{2}$. Les facteurs identifiés qui influencent la déforestation peuvent être regroupés autour de trois éléments principaux de nature démographique, économique et politique.

\subsection{Les facteurs démographiques}

Différentes variables sont utilisées dans les travaux empiriques pour mesurer l'effet des facteurs démographiques : la taille de la population totale, la croissance de la population totale, la densité de population ou encore la part de la population rurale dans la population totale. A partir d'un échantillon de 39 pays d'Afrique, d'Amérique latine et d'Asie sur la période 1968-1978, Allen et

2 Pour une synthèse de la littérature empirique sur la déforestation, se reporter à Angelsen et Kairnmovitz (1999), Barbier (2001), Meyer et alii (2003), Barbier (2004). 
Barnes (1985) montrent qu'une augmentation de la population totale est associée à une réduction des superficies forestières. Utilisant un échantillon de 66 pays sur la période 1972-1991, Bhattarai et Hammig (2001) trouvent que le taux de croissance de la population et la densité de la population rurale ont des effets différents sur la déforestation selon les groupes de pays. L'accroissement de la population est significatif et négatif en Amérique latine et en Afrique, mais positif en Asie. La densité de la population rurale est significative et négative uniquement en Asie.

\subsection{Les facteurs économiques}

Le débat entre croissance économique et déforestation se résume principalement à la mise en évidence d'une relation en forme de U inversé, appelée courbe environnementale de Kuznets (CEK). La CEK a été obtenue pour des indices environnementaux relatifs à la qualité de l'air ou à la qualité de l'eau (Selden et Song, 1994 ; Shafik, 1994 ; Grossman et Krueger, 1995). Les résultats obtenus pour les surfaces forestières sont plus contrastés.

Cropper et Griffiths (1994), ainsi que Bhattarai et Hammig (2001) trouvent une CEK reliant revenu par habitant et taux de déforestation pour les pays africains et les pays d'Amérique latine. Culas (2007), Bhattarai et Hammig (2004) et Combes et alii (2009) mettent en évidence une CEK pour la déforestation en insistant sur l'importance des facteurs institutionnels. En revanche, Shafik (1994) et Koop et Tole (1999) ne trouvent aucune CEK décrivant la relation entre revenu par habitant et déforestation sur des périodes similaires. Azomahou et Nguyen Van (2003) obtiennent même une relation en forme de U entre le revenu par habitant et la déforestation.

D'autres variables économiques sont susceptibles d'influencer la déforestation. Pour la période 1961-1988, Arcand et alii (2008) montrent qu'une dépréciation du taux de change réel accentue la déforestation dans les pays en développement, mais l'atténue dans les pays développés. Le commerce international est aussi l'objet de considération (Barbier, 1994). Par ailleurs, la dette extérieure peut encourager les pays en développement à déforester afin de se procurer des devises étrangères (Rudel et Roper, 1997 ; Bhattarai et Hammig, 2001). L'urbanisation est également reconnue comme un facteur déterminant la déforestation (Ehrhardt-Martinez, 1998). Des travaux empiriques ont aussi montré que les infrastructures jouaient un rôle important dans la déforestation (Nelson et Hellerstein, 1997 ; Rudel et Roper, 1997).

Enfin, des études ont souligné l'impact de la pauvreté et des inégalités sur la déforestation (Ehrhardt-Martinez, 1998 ; Chomitz et alii, 2007 ; Alix-Garcia, 2008). Toutefois, Angelsen et Kairmowitz (1999) précisent qu'il s'agit 
d'un thème de recherche encore inexploré. Les études empiriques adoptant une perspective macroéconomique restent rares sur ce sujet car les données à disposition sont lacunaires ${ }^{3}$.

\subsection{Les facteurs politiques}

D'autres études se sont intéressées à l'influence des facteurs politiques sur la déforestation. Il convient de noter que ces travaux sont cependant moins nombreux que ceux axés sur les facteurs économiques, en partie à cause du manque de données existantes sur le sujet. Deacon (1994), Didia (1997) et Bhattarai et Hammig (2001) soulignent que la déforestation est accentuée dans les pays où la démocratie est faible et les institutions politiques de mauvaise qualité. Par ailleurs, les droits de propriété et les difficultés rencontrées pour les faire respecter dans certains pays en développement peuvent également constituer des facteurs explicatifs de la déforestation (Angelsen, 1999; Deacon, 1999).

\subsection{Quel rôle attendu pour les transferts migratoires ?}

Cette revue de la littérature souligne la diversité des facteurs explicatifs de la déforestation, même s'il n'existe guère de consensus sur le rôle avéré des facteurs sélectionnés, sans doute parce qu'ils agissent conjointement dans le processus de déforestation. Dans le même temps, elle révèle l'absence de considération portée aux questions migratoires. D'un point de vue théorique, les transferts migratoires ont deux effets principaux sur le processus de déforestation. Dans la mesure où ces incidences sont de sens contraire, l'effet final apparait indéterminé.

D'une part, en tant que seconde source de financement extérieur des pays en développement, les transferts migratoires viennent augmenter le revenu national de ces pays et ils contribuent ainsi fortement à la croissance économique. Dans le même temps, les phases du développement économique s'accompagnent d'une augmentation de la population, d'une industrialisation et d'une urbanisation accrues qui viennent peser sur les ressources forestières. Les revenus de l'émigration internationale à destination des pays en développement peuvent, dans ce cadre, contribuer à l'accélération de la déforestation.

3 Par exemple, l'indice de Gini mesurant la dispersion des revenus qui est fourni par la Banque Mondiale en 2008 dans sa base de données World Development Indicators est disponible pour un nombre limité de pays. Sur nos 151 observations, nous n'avons obtenu que 28 observations pour cette variable. Il est de même pour le seuil de pauvreté en pourcentage de la population totale, pour lequel nous n'obtenons que 6 observations. 
D'autre part, les transferts migratoires considérés en part du PIB sont particulièrement élevés à destination des pays à faible revenu et sont reconnus comme un instrument de lutte contre la pauvreté des populations qui en bénéficient. À partir d'un échantillon de 74 pays, Adams et Page (2005) montrent qu'une hausse de $10 \%$ du nombre de migrants internationaux entraîne une baisse de 1,9\% des personnes vivant avec moins d'un dollar par jour. Sur la base d'études microéconomiques pour le Burkina Faso et le Ghana, Lachaud (1999) et Adams (2006) montrent également que les transferts migratoires destinés aux ménages restés au pays d'origine permettent de réduire la pauvreté et son intensité ${ }^{4}$. Or, comme le rappelle la Banque Mondiale (2006), la déforestation est aussi bien le fait des grandes entreprises forestières que des populations pauvres. Dans cette perspective, en faisant reculer la pauvreté des populations locales, les transferts migratoires peuvent contribuer à réduire la déforestation et ils devraient avoir un impact bénéfique sur l'environnement.

\section{DONNÉES ET MODÈLE EMPIRIQUE}

\subsection{Les sources statistiques}

L'échantillon que nous retenons pour l'étude comprend 102 pays. Il couvre une période de quinze ans, allant de 1990 à 2005. La liste complète des pays pris en compte figure en annexe 1. La variable que nous cherchons à expliquer est le taux de déforestation $t d e f_{t}$ à la date $t$ défini de la façon suivante : $t d e f_{t}=$-(superficie forestière $t_{t+1}-$ superficie forestière $_{t}$ ) $/$ superficie forestière $_{t}$

Pour calculer ce taux, nous utilisons des données relatives à la superficie forestière (mesurée en milliers d'hectares) extraites du rapport de la FAO intitulé «Evaluations des ressources forestières mondiales 2005 ». Ce travail statistique est réalisé à des intervalles de cinq ou dix ans, les trois années de référence retenues étant 1990, 2000 et 2005.

Les données de la FAO sont les plus exhaustives actuellement disponibles et font autorité sur le plan international. Ceci peut être illustré à travers deux exemples. D'un côté, les données forestières de la FAO sont utilisées comme indicateur pour la surveillance des progrès vers les objectifs du millénaire pour le développement convenus par les Nations Unies. De l'autre, elles sont utili-

Outre les transferts migratoires, ce sont plus généralement l'ensemble des transferts qui circulent entre les ménages qui vont contribuer à réduire la pauvreté des bénéficiaires (Dimova et Wolff, 2008). 
sées par le Groupe Intergouvernemental d'Experts sur le Changement Climatique (GIEC), qui est chargé par les Nations Unies d'évaluer les conséquences du réchauffement climatique. Toutefois, elles souffrent de limites, notamment en matière de collecte des données car ce sont les gouvernements des pays euxmêmes qui les transmettent à la FAO.

$\mathrm{Au}$ regard de la littérature existante, notre échantillon contient un nombre relativement élevé de pays (102). A titre de comparaison, Koop et Tole (2001) utilisent une base de données comprenant 48 pays. En outre, nous utilisons les dernières statistiques forestières fournies par la FAO en 2005 , ce qui nous permet d'analyser les tendances les plus récentes de la déforestation dans les pays en développement. Certes, nous ne disposons pas d'informations sur la déforestation au cours des années 1970 et 1980, prises en considération par exemple par Azomahou et Nguyen Van (2007), mais il importe d'avoir à l'esprit que les données sur les transferts migratoires pour ces périodes sont pratiquement inexistantes.

A l'instar des études menées à ce jour sur la déforestation, nous retenons les facteurs explicatifs suivants. Afin de mesurer l'incidence des facteurs économiques, nous retenons le PIB par tête (pibt) exprimé en dollars constants avec pour année de référence 2000, et le taux de croissance annuel du PIB exprimé en pourcentage (pibc). Ces données sont extraites des indicateurs de la Banque Mondiale World Development Indicators (WDI, 2008). En outre, nous utilisons une variable relative au taux de change réel exprimé en pourcentage (change). Les données proviennent de la base de données du FMI International Financial Statistics (2008). Enfin, nous retenons une variable d'ouverture commerciale qui correspond aux exportations de biens et services exprimées en pourcentage du PIB (commerce) et une variable de dette extérieure exprimée en pourcentage du PIB (dette). Ces deux variables sont extraites du WDI (2008).

Le rôle potentiel des facteurs démographiques sur la déforestation est pris en compte à travers la densité de la population ( $p o p d)$, exprimée en nombre de personnes par hectare, et le taux de croissance annuel de la population ( en pourcentage. Ces deux variables sont de nouveau extraites du WDI (2008).

Concernant l'impact des institutions, nous utilisons la base de données Freedom House 2007, qui permet de disposer de statistiques internationales institutionnelles sur l'ensemble de la période 1990-2005. Nous construisons un indicateur de qualité des institutions (institution) à partir de deux variables qui portent respectivement sur les droits politiques et les libertés civiles ( « political rights » et " civil liberties »). Ces deux dimensions sont mesurées sur une échelle allant de 1 à 7 , le niveau 1 correspondant à une haute qualité des institutions et 
le niveau 7 à une basse qualité. Nous faisons alors la somme de ces deux variables pour obtenir un indicateur unique (allant de 1 à 14) ${ }^{5}$.

Nos données pour les transferts migratoires sont tirées de la Banque Mondiale (2008). Plus précisément, nous retenons une mesure des transferts migratoires (transfert) qui correspond pour chaque pays à la part des transferts migratoires en pourcentage du PIB. Le Mexique, l'Inde, la Chine, et les Philippines sont les principaux pays destinataires des transferts migratoires en volume. A l'inverse, lorsque la part des transferts migratoires dans le revenu total du pays est prise en compte, ce sont les pays à faible revenu qui sont en tête du classement comme la Moldavie, Tonga, la Guyane ou encore le Lesotho. Les 30 principaux pays receveurs de transferts migratoires en volume et en pourcentage du PIB pour l'année 2005 sont présentés dans l'annexe 2.

Ces données de la Banque Mondiale (2008) sur les transferts migratoires font uniquement référence aux transferts migratoires officiels, c'est-à-dire l'argent que les migrants internationaux versent à leur famille d'origine par des canaux officiels tels que les banques, les services postaux ou les agences de transactions financières internationales. De fait, elles ne prennent pas en compte les sommes qui transitent par des circuits privés et officieux. La Banque Mondiale (2008) considère que les transferts migratoires sont sous-estimés, en particulier pour ceux à destination des pays en développement. En dépit de cette limite, ces données sont les seules dont nous disposons pour avoir une vue d'ensemble des transferts migratoires à l'échelle mondiale.

Afin de comprendre la spécificité des transferts migratoires sur le processus de déforestation, nous prenons en compte deux autres sources de financement extérieur pour les pays en développement, à savoir l'aide au développement et les investissements directs étrangers (en \% du PIB). Ces données sont extraites du WDI (2008). Idéalement, nous aimerions disposer d'une variable d'aide publique pour réduire la déforestation, mais de telles données n'existent pas pour l'échantillon de pays que nous considérons.

Afin de contrôler l'effet de la pauvreté, nous retenons une variable relative à l'espérance de vie, qui est mesurée en nombre d'années. Les données sont extraites du WDI (2008). Comme nous l'avons expliqué précédemment, les données de pauvreté sont pratiquement inexistantes dans les indicateurs fournis par la Banque Mondiale. Par ailleurs, il convient de souligner qu'il peut être pertinent de prendre en compte la superficie forestière initiale dans l'analyse économétrique des déterminants de la déforestation (Arcand et alii, 2008). Nous nous appuyons alors sur les données forestières de la FAO (2005).

5 Azomahou et Nguyen Van (2007) utilisent le même indicateur agrégé pour capter l'effet des institutions politiques sur la déforestation. 


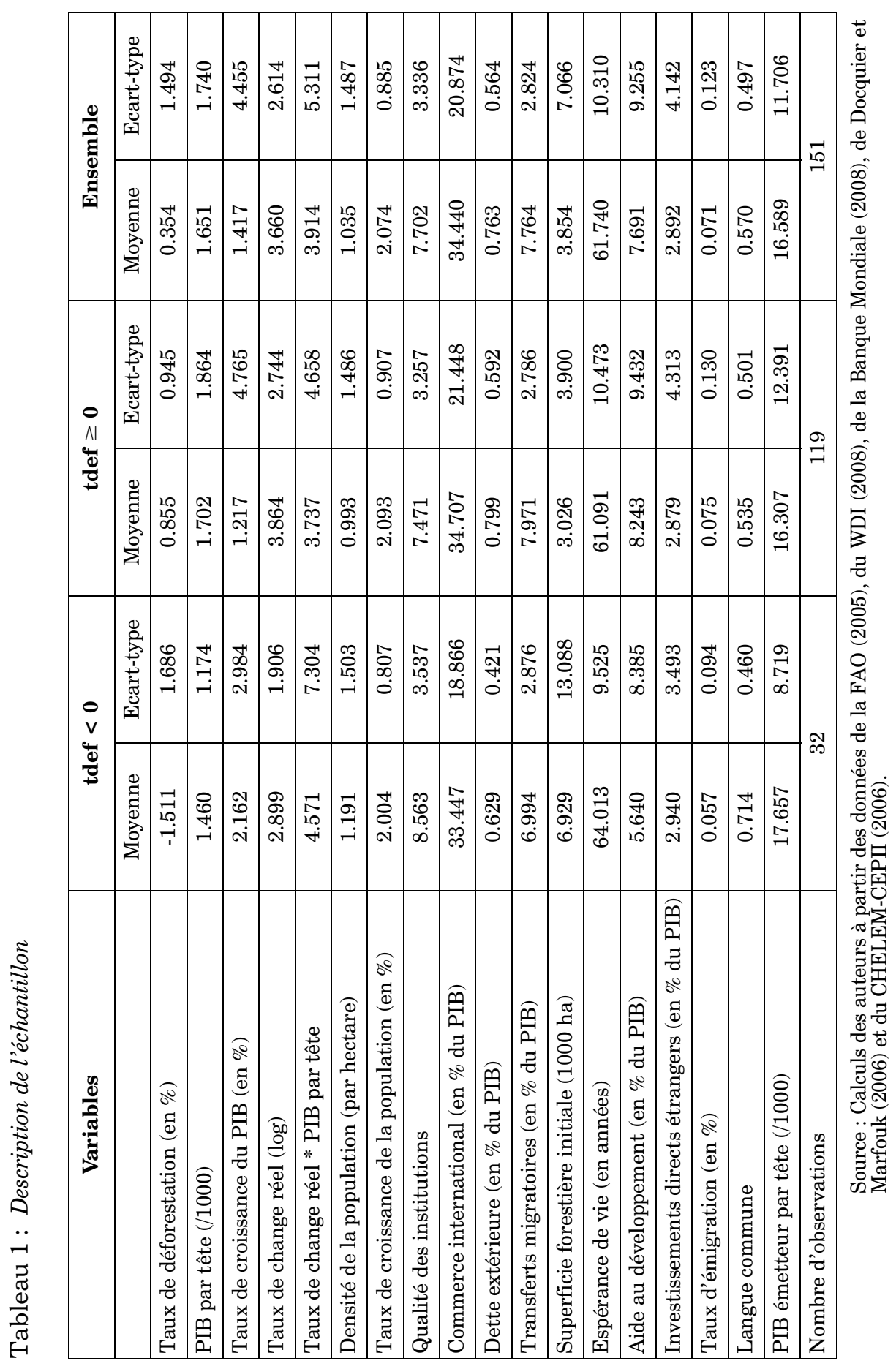




\subsection{Statistiques descriptives}

Les statistiques descriptives pour chacune de ces variables sont reportées dans le tableau 1. La colonne 1 concerne les pays dont le taux de déforestation est négatif (32 observations), tandis que ceux dont le taux de déforestation est positif ou nul (119 observations) sont en colonne 2. L'échantillon complet comprend 151 observations correspondant à 102 pays (échantillon non cylindré).

D'après les données, le taux moyen de déforestation pour l'ensemble des pays s'élève à $0,3 \%$. Pour les pays dont le taux de déforestation est négatif, il s'élève en moyenne à $-1,5 \%$. Pour les pays dont le taux de déforestation est positif, il s'élève en moyenne à $0,8 \%$. Le PIB par tête pour l'ensemble des pays est en moyenne égal à $1651 \$$. Les pays dont le taux de déforestation est négatif ont un PIB par tête inférieur aux pays dont le taux de déforestation est positif (1 $460 \$$ contre $1702 \$$ ). En revanche, le taux de croissance du PIB est le plus élevé dans les pays dont le taux de déforestation est négatif $(2,1 \%$ contre $1,2 \%$ par an).

Concernant les variables démographiques, la densité de population est de 1 personne par hectare pour l'ensemble de l'échantillon. Elle est plus faible dans les pays dont le taux de déforestation est positif $(0,9$ contre 1,1$)$. Le taux de croissance de la population s'élève à $2 \%$ par an pour l'ensemble de l'échantillon. La part des exportations en pourcentage du PIB s'élève à $34,4 \%$ pour l'ensemble de l'échantillon. Ce ratio d'ouverture commerciale est plus élevé dans les pays dont le taux de déforestation est positif $(33,4 \%$ contre $34,7 \%)$. La dette extérieure exprimée en pourcentage du PIB s'élève à $0,7 \%$ pour l'ensemble de l'échantillon. L'espérance de vie pour l'ensemble des pays est en moyenne de 61,7 ans.

Concernant les investissements directs étrangers (en \% du PIB), ce sont les pays qui connaissent un taux de déforestation négatif qui reçoivent le plus $(2,9 \%$ contre $2,8 \%)$. C'est l'inverse pour l'aide au développement $(8,2 \%$ contre $5,6 \%$ ), ainsi que pour les transferts migratoires puisque ce sont les pays qui ont un taux de déforestation positif qui reçoivent relativement plus $(7,9 \%$ contre $6,9 \%$ ). Enfin, les pays qui se caractérisent par un taux de déforestation positif sont ceux qui connaissent le taux d'émigration le plus élevé $(0,07 \%$ contre $0,05 \%)$.

\subsection{Spécification économétrique}

L'étude des déterminants des surfaces forestières nous conduit à exprimer le taux de déforestation en fonction de facteurs à la fois économiques, démogra- 
phiques, institutionnels et migratoires. Nous cherchons à estimer les paramètres de la spécification économétrique suivante :

$t d e f_{i t}=\beta_{0}+\beta_{1}{ }^{*}$ pibt $_{i t}+\beta_{2}{ }^{*}$ pibt $_{i t}{ }^{2}+\beta_{3}{ }^{*}$ pibt $_{i t}{ }^{3}+\beta_{4}{ }^{*} p i b c_{i t}+\beta_{5}{ }^{*}$ change $_{i t}+\beta_{6}{ }^{*}$ popd $_{i t}$ $+\beta_{7}{ }^{*}$ popg $_{i t}+\beta_{8}{ }^{*}$ institution $_{i t}+\beta_{9}{ }^{*}$ commerce $_{i t}+\beta_{10}{ }^{*}$ dette $_{i t}+\beta_{11}{ }^{*}$ transfert $_{i t}+a_{i}+\varepsilon_{i t}$

où les différents $\beta$ sont les paramètres d'intérêt à estimer, les indices $i$ et $t$ indiquant respectivement le pays considéré et l'année d'observation ${ }^{6}$. Nous avons également estimé une variante de (1) comprenant la superficie forestière initiale comme facteur explicatif. Nous retenons un profil quadratique pour le PIB par tête afin de tester l'existence d'une courbe environnementale de Kuznets. Dans l'équation (1), $a_{i}$ et $\varepsilon_{i t}$ sont des termes d'erreurs pour lesquels nous supposons de manière usuelle qu'ils suivent des lois normales et que $E\left[a_{i}\right]=0, E\left[u_{i t}\right]=0, E\left[a_{i}^{2}\right]=\sigma_{a}^{2}, E\left[u_{i t}^{2}\right]=\sigma_{u}^{2}$ et $E\left[a_{i} u_{i t}\right]=0$.

Outre une estimation basique par les Moindres Carrés Ordinaires, nous avons estimé des modèles à effets aléatoires (par les Moindres Carrés Généralisés) et à effets fixes puisque nous disposons d'informations répétées pour un grand nombre de pays. L'hypothèse sous-jacente du modèle à effets aléatoires est que les facteurs explicatifs ne sont pas corrélés avec les termes $a_{i}$ propres à chaque pays. Il est néanmoins possible qu'il existe des caractéristiques propres à chaque pays, non prises en compte dans la régression, corrélées avec les facteurs explicatifs retenus. Dans ce cas, le modèle approprié est un modèle à effets fixes, la régression comportant un ensemble de variables muettes propres à chaque pays dont les $a_{i}$ sont simplement les coefficients estimés des effets fixes pays.

De manière intéressante, les résultats des études empiriques ont généralement été obtenus à partir de modèles à effets fixes. Si le recours à des données annuelles sur des périodes supérieures à 20 ans révèle qu'il importe de tenir compte des sources d'hétérogénéité inobservée entre les pays, la situation est un peu différente dans notre cas puisque nous observons les surfaces forestières à seulement trois dates, si bien que nous pouvons construire seulement deux taux de déforestation. De manière classique, nous avons mis en œuvre des tests du multiplicateur de Breusch et Pagan (1980) et de Hausman (1978) afin de savoir quelle spécification est la plus appropriée pour les données que nous utilisons.

Dans la mesure où la variable dépendante est le taux de déforestation, nous disposons potentiellement de 204 observations pour l'étude empirique (à savoir 102 pays avec deux mesures du taux de déforestation par pays). En raison de données manquantes pour certains pays, nous disposons de 151 observations. 


\section{RÉSULTATS ÉCONOMÉTRIQUES}

\subsection{Les déterminants de la déforestation}

Les résultats sont présentés dans le tableau 2. Dans un premier temps, nous estimons des régressions sans la variable de superficie forestière initiale (colonnes 1 à 5), puis nous introduisons ensuite ce facteur explicatif (colonne 6). Comme l'ont souligné Arcand et alii (2008), le taux de déforestation devrait être corrélé positivement avec la superficie forestière initiale, ce que suggère bien le tableau $1^{7}$. Les estimations sont obtenues par les MCG dans la mesure où le test de Hausman révèle que la spécification à effets fixes n'est pas la plus appropriée pour nos données (au seuil de 5\%). L'hypothèse sous-jacente est donc celle d'une absence de corrélation entre les effets spécifiques pays et les différentes variables explicatives retenues dans la régression.

Tout d'abord, les résultats indiquent que le PIB par tête n'est pas significatif. Il en est de même pour la variable de taux de croissance du PIB. Ces résultats permettent de savoir s'il existe ou non une courbe environnementale de Kuznets reliant le PIB par tête et le taux de déforestation. Les différentes régressions du tableau 2 suggèrent qu'il n'existe pas de CEK pour la déforestation sur la période considérée, de 1990 à 2005. Ceci rejoint les conclusions des travaux de Koop et Tole (1999) et Azomahou et Nguyen Van (2003, 2007) et la CEK semble donc s'observer davantage pour des indices environnementaux qualitatifs tels la qualité de l'eau ou la qualité de l'air (Shafik, 1994 ; Selden et Song, 1994) que pour les surfaces forestières.

Ensuite, les résultats relatifs aux variables démographiques apparaissent non significatifs. En revanche, le taux de change réel tend à accélérer le processus de déforestation dans les pays en développement comme l'ont montré Arcand et alii (2008). De même, les résultats relatifs à la qualité des institutions semblent plus robustes et une bonne qualité des institutions vient atténuer le processus de déforestation (Deacon, 1994 ; Didia, 1997 ; Bhattarai et Hammig, 2001).

7 Par exemple, le Brésil est le pays au monde qui possède la superficie forestière la plus élevée et qui connaît la déforestation la plus élevée sur la période 2000-2005 (perte annuelle de 3103 milliers d'hectares) (FAO, 2005). 
Tableau 2: Les déterminants de la déforestation

\begin{tabular}{|c|c|c|c|c|c|c|}
\hline Variables & (1) & (2) & (3) & (4) & (5) & (6) \\
\hline \multirow{2}{*}{ Constante } & 1.079 & 1.112 & 0.211 & 1.303 & 1.051 & 0.286 \\
\hline & $\begin{array}{l}(1.34) \\
\end{array}$ & $\begin{array}{l}(1.38) \\
\end{array}$ & $\begin{array}{l}(0.17) \\
\end{array}$ & $\begin{array}{l}(1.58) \\
\end{array}$ & $\begin{array}{l}(1.25) \\
\end{array}$ & $\begin{array}{l}(0.28) \\
\end{array}$ \\
\hline \multirow{2}{*}{ PIB par tête } & -0.722 & -0.766 & -0.850 & -0.881 & -0.710 & -0.628 \\
\hline & $(1.36)$ & $(1.46)$ & $(1.40)$ & $(1.62)$ & $(1.33)$ & $(1.18)$ \\
\hline \multirow{2}{*}{ PIB par tête ${ }^{2}(/ 1000)$} & 2.074 & 2.137 & 2.467 & $2.438^{*}$ & 2.042 & 1.882 \\
\hline & $(1.44)$ & $(1.49)$ & $(1.52)$ & $(1.66)$ & $(1.40)$ & $(1.31)$ \\
\hline \multirow{2}{*}{$\begin{array}{l}\begin{array}{l}\text { PIB par tête } \\
(/ 100000)\end{array} \\
\end{array}$} & -1.533 & -1.558 & -1.858 & -1.790 & -1.513 & -1.369 \\
\hline & $(1.32)$ & $(1.35)$ & $(1.45)$ & $(1.52)$ & $(1.30)$ & $(1.19)$ \\
\hline \multirow{2}{*}{$\begin{array}{l}\text { Taux de croissance du } \\
\text { PIB }\end{array}$} & -0.017 & & -0.023 & -0.020 & -0.017 & -0.019 \\
\hline & $(0.74)$ & & $(0.94)$ & $(0.89)$ & $(0.74)$ & $(0.82)$ \\
\hline \multirow{2}{*}{ Taux de change réel } & $0.191^{* *}$ & $0.181^{* *}$ & $0.207^{* * *}$ & $0.181^{* *}$ & $0.192^{* * *}$ & $0.195^{* *}$ \\
\hline & $(2.44)$ & $(2.34)$ & $(2.54)$ & $(2.30)$ & $(2.43)$ & $(2.46)$ \\
\hline \multirow{2}{*}{$\begin{array}{l}\text { Taux de change réel * } \\
\text { PIB par tête }\end{array}$} & -0.049 & -0.045 & -0.055 & -0.048 & -0.049 & -0.058 \\
\hline & $(1.35)$ & $(1.26)$ & $(1.48)$ & $(1.33)$ & $(1.35)$ & $(1.55)$ \\
\hline \multirow{2}{*}{\begin{tabular}{|l|}
$\begin{array}{l}\text { Densité de la } \\
\text { population }\end{array}$ \\
\end{tabular}} & 0.069 & 0.063 & 0.060 & 0.061 & 0.069 & 0.119 \\
\hline & $(0.81)$ & $(0.74)$ & $(0.70)$ & $(0.72)$ & $(0.81)$ & $(1.24)$ \\
\hline \multirow{2}{*}{$\begin{array}{l}\text { Taux de croissance } \\
\text { de la population }\end{array}$} & -0.073 & -0.061 & -0.067 & -0.072 & -0.071 & -0.083 \\
\hline & $(0.55)$ & $(0.47)$ & $(0.49)$ & $(0.54)$ & $(0.53)$ & $(0.63)$ \\
\hline \multirow{2}{*}{\begin{tabular}{|l|} 
Qualité des \\
institutions
\end{tabular}} & $-0.079 * *$ & $-0.077^{* *}$ & $-0.077^{* *}$ & $-0.080 * *$ & $-0.078^{* *}$ & $-0.073^{* *}$ \\
\hline & $(2.13)$ & $(2.08)$ & $(2.01)$ & $(2.16)$ & $(2.04)$ & $(1.97)$ \\
\hline \multirow{2}{*}{$\begin{array}{l}\text { Commerce } \\
\text { international }\end{array}$} & 0.004 & 0.003 & 0.002 & 0.003 & 0.003 & 0.005 \\
\hline & $(0.58)$ & $(0.52)$ & $(0.35)$ & $(0.53)$ & $(0.55)$ & $(0.78)$ \\
\hline \multirow{2}{*}{ Dette extérieure } & -0.011 & -0.014 & 0.054 & 0.129 & -0.010 & 0.012 \\
\hline & $(0.05)$ & $(0.06)$ & $(0.21)$ & $(0.48)$ & $(0.04)$ & $(0.05)$ \\
\hline \multirow{2}{*}{ Transferts migratoires } & $-0.051^{* * *}$ & $-0.053^{* * *}$ & $-0.054^{* * *}$ & $-0.047 * * *$ & $-0.051 * * *$ & $-0.042^{* *}$ \\
\hline & $(3.19)$ & $(3.35)$ & $(3.24)$ & $(2.87)$ & $(3.16)$ & $\begin{array}{l}(2.37) \\
\end{array}$ \\
\hline \multirow{2}{*}{ Espérance de vie } & & & 0.015 & & & \\
\hline & & & $(0.85)$ & & & \\
\hline \multirow{2}{*}{ Aide au développement } & & & & -0.020 & & \\
\hline & & & & $(1.27)$ & & \\
\hline \multirow{2}{*}{$\begin{array}{l}\text { Investissements } \\
\text { directs étrangers }\end{array}$} & & & & & 0.003 & \\
\hline & & & & & $(0.10)$ & \\
\hline \multirow{2}{*}{\begin{tabular}{|l}
$\begin{array}{l}\text { Superficie forestière } \\
\text { initiale }\end{array}$ \\
\end{tabular}} & & & & & & 0.071 \\
\hline & & & & & & $(1.17)$ \\
\hline \multicolumn{7}{|l|}{$\begin{array}{l}\text { Test de Breusch } \\
\text { et Pagan }\end{array}$} \\
\hline Chi-2 ; d.l. ; prob. & $\begin{array}{l}31.20 ; 1 ; \\
0.000\end{array}$ & $\begin{array}{l}32.29 ; 1 \\
0.000\end{array}$ & $\begin{array}{l}30.09 ; 1 ; \\
0.000\end{array}$ & $\begin{array}{l}29.31 ; 1 \\
0.000\end{array}$ & $\begin{array}{l}29.56 ; 1 \\
0.000\end{array}$ & $\begin{array}{l}61.56 ; 1 \\
0.000\end{array}$ \\
\hline \multicolumn{7}{|l|}{ Test de Hausman } \\
\hline Chi-2 ; d.l. ; prob. & $\begin{array}{l}14.87 ; 12 \\
0.249\end{array}$ & $\begin{array}{l}14.02 ; 11 \\
0.523\end{array}$ & $\begin{array}{l}14.23 ; 13 \\
0.358\end{array}$ & $\begin{array}{l}16.25 ; 13 \\
0.236\end{array}$ & $\begin{array}{l}19.41 ; 13 \\
0.111\end{array}$ & $\begin{array}{l}19.94 ; 13 ; \\
0.097\end{array}$ \\
\hline Nombre d'observations & 151 & 151 & 144 & 151 & 151 & 151 \\
\hline Nombre de pays & 88 & 88 & 85 & 88 & 88 & 88 \\
\hline
\end{tabular}

Notes: Les estimateurs sont obtenus par la méthode des Moindres Carrés Généralisés (modèles à effets aléatoires). Les valeurs absolues des t de Student sont entre parenthèses sous les coefficients. Les seuils de significativité retenus sont respectivement de $1 \%\left({ }^{* * *}\right), 5 \%\left(^{* *}\right)$ et $10 \%\left(^{*}\right)$. 
Les données révèlent qu'il est assez difficile d'expliquer le taux de déforestation sur la période considérée. Conformément à d'autres travaux sur la déforestation, en particulier Koop et Tole (1999) et Azomahou et Nguyen Van (2007), les variables explicatives sont rarement significatives. La méthode d'estimation retenue importe d'ailleurs peu pour les résultats. Par exemple, les variables de commerce international et de dette extérieure ne sont jamais significatives dans les différentes régressions. Une explication peut venir du nombre limité d'observations.

Nous nous intéressons à présent au rôle des transferts migratoires. En colonne 1 du tableau 2 , nous ajoutons aux variables explicatives précédentes la réception de transferts migratoires (en pourcentage du PIB). Nous excluons ensuite le taux de croissance du PIB (colonne 2). Son introduction en colonne 1 comme variable de contrôle signifie que nous neutralisons l'impact des transferts migratoires passant par la stimulation de la croissance. En colonne 3, nous adoptons une démarche similaire pour la pauvreté, mesurée à travers l'espérance de vie. Dans les colonnes 4 et 5 , nous tentons de tenir compte de la spécificité des transferts migratoires comme source de financement extérieur dans le processus de déforestation en introduisant la variable explicative d'aide au développement, puis celle d'IDE.

Alors que nous avons souligné la difficulté d'expliquer la déforestation dans la littérature empirique antérieure, nous trouvons un coefficient négatif et significatif au seuil de $1 \%$ pour la variable de transferts migratoires reçus. Le processus de déforestation apparaît donc atténué lorsque le poids des transferts migratoires reçus dans le revenu national s'accroît. Cet effet négatif des transferts migratoires reste vérifié lorsque le taux de croissance du PIB n'est pas pris en compte (colonne 2) ou bien lorsque l'on contrôle par la pauvreté (colonne 3). Par ailleurs, si les transferts migratoires viennent dans tous les cas freiner la déforestation, l'aide au développement n'a en revanche aucun effet environnemental (colonne 4) et il en est de même pour les IDE (colonne 5).

Plusieurs explications sont possibles pour comprendre le rôle des transferts migratoires. Dans une perspective microéconomique, il est reconnu que les transferts migratoires représentent un élément important d'amélioration des conditions de vie. Ils remplissent une fonction d'assurance et sont un instrument de lutte contre la pauvreté (Gubert, 2005 ; Adams et Page, 2005). Il est vraisemblable que les transferts migratoires permettent une gestion plus raisonnée des surfaces forestières pour les populations rurales et pauvres.

L'utilisation des transferts migratoires est variée pour ceux qui en bénéficient (Osili, 2004 ; Gubert, 2005). En majorité, ils permettent de subvenir aux 
besoins de première nécessité et d'améliorer le logement. Une partie est également affectée à des investissements en capital humain qui influent sur le développement à long terme (McKenzie et Rapoport, 2007). La réception de transferts migratoires permet aussi de recourir à des investissements dans du matériel agricole (Gubert, 2005). Avec une agriculture plus intensive sur les terres, la pression sur les superficies forestières est réduite. Enfin, les ménages bénéficiaires de transferts migratoires peuvent accéder dans la légalité à des terres. Ces droits de propriété peuvent leur éviter de dégrader les forêts, qui sont considérées dans la plupart des pays en développement comme un bien public (Deacon, 1994).

Dans une perspective macroéconomique, les flux financiers de l'émigration internationale permettent aux pays en développement d'importer plus de biens agricoles des autres pays. Cela vient réduire la conversion des forêts en terres arables, ce processus étant reconnu comme un facteur important de la déforestation (Angelsen, 1999). Par ailleurs, les transferts migratoires reçus peuvent être interprétés comme un signal de la réussite de la migration. Ceci va influencer non seulement les flux de population entre les pays, avec par exemple une émigration accrue qui peut réduire les problèmes posés par la forte pression démographique, mais aussi au sein même des pays avec des transitions accrues entre les milieux ruraux et urbains.

D'après nos résultats, les transferts migratoires semblent bien jouer un rôle spécifique dans le processus de déforestation, puisque l'aide au développement et les IDE n'avaient aucune incidence significative sur la déforestation ${ }^{8}$. Une première explication à la spécificité des transferts migratoires dans le processus de déforestation vient de ce qu'ils constituent un apport financier plus stable que les autres sources de financement extérieur à destination des pays en développement (Gammeltoft, 2002). Ils sont qualifiés à ce titre de contracycliques par certains auteurs (Ratha, 2005), c'est-à-dire qu'ils augmentent pendant des périodes de ralentissement économique ou après une catastrophe naturelle, contrairement aux IDE qui diminuent au cours de ces périodes ${ }^{9}$.

Une seconde explication vient de ce que l'instabilité macroéconomique peut être un facteur de déforestation (Arcand et alii, 2008). En effet, les chocs

$8 \mathrm{Au}$ cours des dernières années, les transferts migratoires sont devenus la deuxième source de financement extérieur pour les pays en développement. Pour certains pays, les transferts migratoires sont même supérieurs aux IDE. A titre d'exemple, les transferts migratoires à destination des Philippines représentent 14 milliards de dollars en 2005, tandis que les IDE sont estimés à 2 milliards de dollars (Banque Mondiale, 2008).

9 Toutefois, le débat reste ouvert dans la littérature sur le caractère contracyclique des transferts migratoires (Chami et alii, 2003; Ratha, 2005; Yang, 2008). 
macroéconomiques affectent fortement les revenus des populations dans les pays en développement. Celles-ci vont alors augmenter la pression sur les ressources naturelles. La variable d'IDE prise en compte dans la régression permet de contrôler pour l'instabilité macroéconomique. En comparant le coefficient estimé de la variable de transferts migratoires avec et sans contrôle par l'instabilité macroéconomique, nous constatons que le coefficient estimé des transferts migratoires est négatif (et significatif) dans les deux cas. Cela pourrait mettre en évidence le rôle contracyclique des transferts migratoires, qui joueraient un rôle d'assurance favorable aux superficies forestières.

Une troisième explication est que, contrairement à l'aide au développement, les transferts migratoires ne passent pas par le budget de l'Etat. Nous pouvons donc supposer qu'ils ne peuvent pas être détournés par les gouvernements nationaux. En outre, ils sont plus ciblés. Un trait caractéristique des transferts migratoires par rapport aux IDE et à l'aide au développement est leur nature personnelle. Les transferts migratoires sont destinés à des familles précises dans les pays d'origine des migrants internationaux (Gubert, 2005). Ils constituent une source supplémentaire de revenus pour ceux qui en bénéficient et, en ce sens, peuvent directement agir sur leurs décisions.

Les transferts migratoires possèdent donc certains traits caractéristiques qui les distinguent des autres sources de financement extérieur à destination des pays en développement. D'un côté, ces transferts agissent directement sur les ménages qui en bénéficient. De l'autre, ils sont redistributifs dans la mesure où les migrants versent de l'argent aux membres défavorisés de leurs familles qui sont restés au pays d'origine. En luttant contre la pauvreté des populations dépendantes des ressources forestières, les transferts migratoires limitent les atteintes faites aux forêts et les investissements permis par ces flux conduisent à une gestion plus raisonnée des terres.

Pour finir, nous avons introduit dans la colonne (6) la superficie forestière initiale dans la régression. Les estimateurs du modèle à effets aléatoires mettent en évidence une corrélation positive entre le taux de déforestation et la couverture forestière initiale, mais cette relation n'apparaît pas statistiquement significative. Toutefois, pour cette spécification, le test de Hausman suggère que le modèle à effets aléatoires n'est pas forcément le plus approprié, puisque la statistique correspondante est significative au seuil de $9,7 \%$. Nous avons donc également estimé un modèle à effets fixes pour cette spécification.

Les résultats, non reportés, mettent en évidence un effet positif de la surface initiale, statistiquement significatif (avec un t de 2,32). En revanche, plus aucune autre variable explicative ne vient influencer le taux de déforestation. Une difficulté vient assurément de la faible variabilité temporelle de nos don- 
nées, puisque nous avons au plus deux observations par pays. L'introduction de la surface forestière initiale donne lieu au problème classique de condition initiale qui se pose dans l'estimation de modèles de panel dynamiques, mais la trop faible variabilité temporelle des données ne nous permet pas d'explorer cet aspect contrairement à d'autres auteurs qui utilisent des séries beaucoup plus longues sur la déforestation (Arcand et alii, 2008). Nous avons donc choisi dans la suite de notre étude empirique de ne pas introduire la surface forestière initiale dans les régressions.

\subsection{Transferts migratoires et endogénéité}

Nous cherchons à présent à savoir si nos résultats sont ou non robustes à la question de l'endogénéité, la corrélation entre déforestation et transferts migratoires pouvant ne pas correspondre à une relation causale. Cette endogénéité peut a prori provenir d'un biais de simultanéité, de variables omises si la déforestation et les transferts migratoires sont déterminés par des caractéristiques communes et difficiles à prendre en compte (néanmoins, les régressions précédentes incluent des effets pays spécifiques), ou encore d'erreurs de mesure sur une variable explicative. Nous avons dès lors recours à la méthode des variables instrumentales pour approfondir ce point.

La principale difficulté à laquelle nous avons été confrontés pour l'analyse empirique consiste à trouver des instruments pertinents, d'autant que la littérature antérieure sur les transferts migratoires a fort peu utilisé cette méthode. Les instruments retenus doivent remplir deux conditions. Ils doivent être (fortement) corrélés à la variable supposée endogène, mais ils ne doivent pas être corrélés avec le terme d'erreur de l'équation principale. La seconde condition, relative à l'exogénéité de l'instrument, peut être testée si l'on dispose d'au moins deux instruments par la mise en œuvre du test de sur-identification de Sargan. Nous avons dès lors procédé de la façon suivante.

Le premier instrument que nous utilisons est le taux d'émigration. Celui-ci est obtenu à partir de la base de données préparée par Docquier et Marfouk (2006), qui fournit des taux d'émigration par niveau de qualification pour les années 1990 et 2000 . Nous sélectionnons alors le taux d'émigration total en 2000. Il est acquis qu'un taux d'émigration élevé dans un pays influence positivement et fortement les transferts migratoires à destination de ce pays (Lianos, 1997 ; Aydas et alii, 2005). Cet instrument fait donc référence au potentiel économique des migrants.

Le second instrument que nous utilisons est le partage d'une langue entre le pays d'émigration $i$ et le pays d'immigration $j$. Pour ce faire, nous nous appuyons tout d'abord sur le Recueil de statistiques sur les migrations et les 
envois de fonds de la Banque Mondiale (2008), qui identifie pour chaque pays $\mathrm{du}$ monde son principal pays d'immigration. Nous le reportons dans notre base de données. Puis, nous utilisons la base de données du CHELEM-CEPII (2006) sur la distance, qui propose une variable bilatérale de langue commune. Il s'agit d'une variable muette indiquant si le pays d'émigration et le pays d'immigration partagent une langue commune. Le partage d'une langue commune devrait être fortement corrélé aux transferts migratoires, en facilitant la constitution de réseaux de migrants internationaux.

Enfin, le troisième instrument que nous utilisons est le PIB par tête du pays d'immigration $j$, qui va nous permettre de contrôler pour la conjoncture économique du pays d'accueil des migrants. Comme précédemment, nous nous appuyons sur le Recueil de statistiques sur les migrations et les envois de fonds de la Banque Mondiale (2008), qui identifie pour chaque pays du monde son principal pays d'immigration. Puis, nous utilisons les données de PIB par tête du WDI (2008). Nous pensons que cet instrument constitue une bonne approximation du potentiel de richesse du pays d'accueil. L'idée est que le PIB par tête du pays émetteur des transferts migratoires va permettre des envois de fonds plus importants vers les pays d'origine.

Contrairement à cette variable de PIB, les deux premiers instruments présentent peu de variabilité temporelle. Au regard de notre étude, ceci ne semble pas trop problématique dans la mesure où la variabilité temporelle de notre échantillon est elle-même faible, avec seulement deux taux de déforestation. Nous appliquons d'abord la méthode des variables instrumentales sans prise en compte de l'hétérogénéité inobservée invariante au niveau pays (IV), puis la méthode des doubles moindres carrés généralisés (2MCG). Les résultats sont présentés dans le tableau 3.

Dans les colonnes 1 et 2 , le seul instrument utilisé pour expliquer les transferts migratoires est le taux d'émigration. Avec ou sans contrôle de l'hétérogénéité inobservée, nous trouvons un signe négatif pour la variable de transferts migratoires, mais la relation n'est pas significative aux seuils habituellement retenus ${ }^{10}$. Dans les colonnes 3 et 4 , nous prenons également en compte l'instrument relatif à la langue commune. Le coefficient estimé pour la variable de transferts migratoires devient alors significatif, même si le seuil n'est que de $10 \%$ en présence d'un modèle à effets aléatoires. Dans les deux cas, une part accrue des transferts migratoires vient réduire la déforestation et l'impact des transferts apparait bien causal. Les valeurs obtenues pour le test d'exclusion des instruments et pour le test de sur-identification de Sargan indiquent par ailleurs que les instruments retenus ont les bonnes propriétés.

${ }^{10}$ Les autres résultats sont conformes à ceux mis en évidence dans le tableau 2. 
Tableau 3 : Régressions instrumentales de l'effet des transferts migratoires

\begin{tabular}{|c|c|c|c|c|c|c|}
\hline \multirow[t]{2}{*}{ Variables } & \multicolumn{2}{|c|}{$\begin{array}{c}\text { Instrument : } \\
\text { taux d'émigration }\end{array}$} & \multicolumn{2}{|c|}{\begin{tabular}{|c|} 
Instrument : \\
taux d'émigration + \\
langue commune
\end{tabular}} & \multicolumn{2}{|c|}{$\begin{array}{c}\text { Instruments : } \\
\text { taux d'émigration }+ \\
\text { langue commune }+ \\
\text { PIB émetteur par tête }\end{array}$} \\
\hline & (1) & (2) & (3) & (4) & (5) & (6) \\
\hline & IV & $2 \mathrm{MCG}$ & IV & $2 \mathrm{MCG}$ & IV & $2 \mathrm{MCG}$ \\
\hline \multirow{2}{*}{ Constante } & 1.891 & 1.446 & $2.574 * *$ & $1.902 *$ & $2.551^{* *}$ & 1.756 \\
\hline & $(1.50)$ & $(1.18)$ & $(2.26)$ & $(1.65)$ & $(2.22)$ & $(1.53)$ \\
\hline \multirow{2}{*}{ PIB par tête } & $-1.196^{* *}$ & -0.847 & $-1.386^{* *}$ & -0.935 & $-1.378^{* *}$ & -0.920 \\
\hline & $(2.26)$ & $(1.49)$ & $(2.50)$ & $(1.53)$ & $(2.47)$ & $(1.53)$ \\
\hline \multirow{2}{*}{$\begin{array}{l}\text { PIB par tête } \\
(/ 1000)\end{array}$} & $3.380^{* *}$ & 2.289 & $3.808^{* *}$ & 2.763 & $3.788^{* *}$ & 2.816 \\
\hline & $(2.36)$ & $(1.57)$ & $(2.24)$ & $(1.52)$ & $(2.22)$ & $(1.57)$ \\
\hline \multirow{2}{*}{$\begin{array}{l}\text { PIB par tête }{ }^{3} \\
(/ 100000)\end{array}$} & $-2.559^{* *}$ & -1.664 & $-2.918^{*}$ & -2.258 & $-2.902^{*}$ & -2.322 \\
\hline & $(2.06)$ & $(1.42)$ & $(1.85)$ & (1.35) & $(1.83)$ & $(1.40)$ \\
\hline \multirow{2}{*}{$\begin{array}{l}\text { Taux de croissance } \\
\text { du PIB }\end{array}$} & -0.029 & -0.014 & -0.028 & -0.013 & -0.024 & -0.011 \\
\hline & $(1.02)$ & $(0.50)$ & $(0.91)$ & $(0.45)$ & $(0.75)$ & $(0.36)$ \\
\hline \multirow{2}{*}{ Taux de change réel } & $0.174^{* *}$ & $0.171^{*}$ & $0.148^{*}$ & 0.156 & $0.149^{*}$ & $0.169 *$ \\
\hline & $(2.06)$ & $(1.74)$ & $(1.80)$ & $(1.57)$ & $(1.81)$ & $(1.73)$ \\
\hline \multirow{2}{*}{$\begin{array}{l}\text { Taux de change réel } \\
\text { * PIB par tête }\end{array}$} & -0.048 & -0.045 & -0.054 & -0.058 & -0.054 & -0.060 \\
\hline & $(1.40)$ & $(1.18)$ & $(1.42)$ & (1.31) & $(1.42)$ & (1.39) \\
\hline \multirow{2}{*}{$\begin{array}{l}\text { Densité de la } \\
\text { population }\end{array}$} & 0.063 & 0.064 & 0.083 & 0.086 & 0.083 & 0.088 \\
\hline & $(0.84)$ & $(0.76)$ & $(1.07)$ & $(0.94)$ & $(1.07)$ & $(0.99)$ \\
\hline \multirow{2}{*}{$\begin{array}{l}\text { Taux de croissance } \\
\text { de la population }\end{array}$} & -0.031 & -0.092 & -0.106 & -0.178 & -0.105 & -0.160 \\
\hline & $(0.16)$ & $(0.57)$ & $(0.56)$ & $(1.06)$ & $(0.55)$ & $(0.95)$ \\
\hline \multirow{2}{*}{$\begin{array}{l}\text { Qualité des } \\
\text { institutions }\end{array}$} & $-0.127^{* * * *}$ & $-0.084 * *$ & $-0.148^{* * *}$ & $-0.090^{* *}$ & $-0.148^{* * *}$ & $-0.092^{* * *}$ \\
\hline & $(3.40)$ & $(2.24)$ & (3.68) & (2.19) & (3.65) & $(2.27)$ \\
\hline \multirow{2}{*}{\begin{tabular}{|l|} 
Commerce \\
international
\end{tabular}} & 0.005 & 0.004 & 0.007 & 0.004 & 0.007 & 0.003 \\
\hline & $(0.83)$ & $(0.59)$ & (1.18) & $(0.54)$ & $(1.14)$ & $(0.51)$ \\
\hline \multirow{2}{*}{ Dette extérieure } & -0.068 & -0.053 & -0.101 & -0.055 & -0.096 & -0.030 \\
\hline & $(0.28)$ & $(0.20)$ & $(0.42)$ & $(0.20)$ & (0.39) & $(0.11)$ \\
\hline \multirow{2}{*}{$\begin{array}{l}\text { Transferts } \\
\text { migratoires }\end{array}$} & -0.085 & -0.072 & $-0.113^{* *}$ & $-0.091^{*}$ & $-0.113^{* *}$ & -0.083 \\
\hline & (1.37) & (1.17) & $(2.16)$ & (1.67) & $(2.16)$ & $(1.55)$ \\
\hline $\begin{array}{l}\mathrm{R}^{2} \text { partiel des } \\
\text { instruments }\end{array}$ & 0,065 & & 0,096 & & 0,097 & \\
\hline \multicolumn{7}{|c|}{\begin{tabular}{|l} 
Test d'exclusion des instruments \\
\end{tabular}} \\
\hline $\mathrm{F} ;$ prob. & 9,$53 ; 0,002$ & & 6,$82 ; 0,001$ & & 4,$49 ; 0,005$ & \\
\hline \multicolumn{7}{|l|}{ Test de Sargan } \\
\hline Chi-2; prob. & - & & 2,$14 ; 0,144$ & & 2,$16 ; 0,142$ & \\
\hline $\begin{array}{l}\text { Nombre } \\
\text { d'observations }\end{array}$ & 151 & 151 & 142 & 142 & 141 & 141 \\
\hline Nombre de pays & 88 & 88 & 83 & 83 & 82 & 82 \\
\hline
\end{tabular}

Notes: Les valeurs absolues des t de Student sont entre parenthèses sous les coefficients. Les seuils de significativité retenus sont respectivement de $1 \%\left(^{* * *}\right)$, $5 \%\left({ }^{* *}\right)$ et $10 \%\left({ }^{*}\right)$. 
Dans un dernier temps, nous ajoutons aux deux instruments précédents le PIB par tête du principal pays d'immigration. Cette dernière variable doit permettre de mieux capturer l'incidence des conditions économiques dans les pays d'accueil, mais le niveau de richesse retenu concerne seulement le pays où les migrants sont les plus nombreux. Pour les deux régressions (IV et 2MCG), nous obtenons de nouveau le signe négatif pour la variable de transferts migratoires, qui viennent donc ralentir le processus de déforestation. Néanmoins, le coefficient estimé de la variable de transferts migratoires est seulement significatif par la méthode des variables instrumentales. Le seuil n'est que de $12,2 \%$ pour le modèle à effets aléatoires une fois l'endogénéité de la variable de transfert prise en compte.

En l'état, ces résultats doivent donc être interprétés avec prudence même si toutes les régressions mises en œuvre vont dans le sens d'un effet réducteur des transferts migratoires sur la déforestation. D'un point de vue empirique, le manque de variabilité temporelle de notre échantillon ou bien encore la difficulté de trouver des instruments pour les transferts migratoires sont autant de limites qu'il convient de garder à l'esprit.

\section{CONCLUSION}

Dans cet article, nous avons cherché à étudier le rôle des transferts migratoires sur le processus de déforestation. S'il est désormais avéré que les transferts migratoires ont des conséquences majeures en termes de santé ou bien d'éducation pour les populations qui en bénéficient, leurs possibles conséquences environnementales ont été ignorées à ce jour. Dans la mesure où ces flux d'argent viennent d'une part contribuer au développement économique des pays et d'autre part réduire la pauvreté des ménages, il est légitime de penser que ces transferts vont avoir une incidence sur l'environnement.

A l'aide de données portant sur 102 pays en développement au cours de la période 1990-2005, nous avons ici examiné le rôle des transferts migratoires sur la réduction des surfaces forestières. Notre étude économétrique met en évidence trois résultats principaux. Tout d'abord, une courbe environnementale de Kuznets reliant PIB par tête et taux de déforestation n'est pas mise en évidence sur l'échantillon considéré. Ensuite, la qualité des institutions tend à freiner la déforestation, tandis que les flux financiers internationaux n'influencent pas significativement la réduction des superficies forestières. Enfin, le taux de déforestation décroît pour l'ensemble des pays au fur et à mesure que la part des transferts migratoires dans le PIB augmente. Cet effet des transferts migratoires sur la réduction des superficies forestières est 
d'importance car la déforestation est reconnue comme étant un problème environnemental majeur pour les pays en développement.

L'approche agrégée que nous avons retenue pour cette étude ne nous permet guère d'explorer plus en détail les mécanismes sous-jacents à cette relation. Les transferts migratoires peuvent permettre d'accroître la productivité agricole par l'achat de nouvelles machines ou bien d'accroître les importations de biens alimentaires, ce qui réduit la pression sur les terres. Parallèlement, ces flux de revenus peuvent aussi donner lieu à des migrations entre les zones rurales et urbaines des pays, ce qui affecte la pression démographique et donc la pression sur les forêts. Il est fort difficile de savoir quels sont les mécanismes à l'œuvre sur la base de données macroéconomiques.

Il convient pour finir de souligner un certain nombre de limites à notre étude. Tout d'abord, la période retenue pour l'étude, de 1990 à 2005, ne permet guère de prendre en considération des éléments de long terme dans l'analyse. La difficulté essentielle vient ici de la mesure des transferts migratoires, pour lesquels les chercheurs disposent de données seulement sur la période récente. Ensuite, il s'avère difficile de trouver des facteurs explicatifs significatifs pour les taux de déforestation, même si ce constat n'est pas propre à notre étude. Enfin, l'absence de courbe environnementale de Kuznets mérite réflexion. Pourquoi le profil en U inversé observé entre le revenu par tête et des indicateurs de qualité environnementale ne s'applique-t-il pas pour la déforestation?

En l'état, notre travail a permis de montrer que les transferts migratoires et l'environnement n'étaient pas deux éléments disjoints. Afin de mieux comprendre l'effet de la migration internationale et des flux financiers qui y sont associés, il serait assurément pertinent de procéder à des analyses économétriques complémentaires sur des pays peu développés, sur la base de données microéconomiques, afin de savoir comment la réception de transferts migratoires vient modifier l'usage des terres agricoles et plus généralement l'allocation des ressources au sein des ménages.

\section{RÉFÉRENCES}

ADAMS R.C. (2006), "Remittances and poverty in Ghana", World Bank Policy Research Working Paper, 3838.

ADAMS R.C., PAGE J. (2005), "Do international migration and remittances reduce poverty in developing countries?", World Development, 33, 1645-1669.

ALIX-GARCIA J. (2008), "An exploration of the positive effect of inequality on common property forests", Journal of Development Economics, 87, 92-105. 
ALLEN J.C., BARNES D.F. (1985), "The causes of deforestation in developing countries", Annals of the Association of American Geographers, 75, 163-184.

ANGELSEN A. (1999), "Agricultural expansion and deforestation: modeling the impact of population, market forces and property rights", Journal of Development Economics, 58, 185-218.

ANGELSEN A., KAIRNMOVITZ D. (1999), "Rethinking the causes of deforestation: Lessons from economic models", World Bank Research Observer, 14, 73-98.

ARCAND J.L., GUILLAUMONT P., GUILLAUMONT JEANNENEY S. (2008), "Deforestation and the real exchange rate", Journal of Development Economics, 84, 242-262.

AYDAS O.T., METIN-OZCAN K., NEYAPTI B. (2005), "Determinants of worker remittances: The case of Turkey", Emerging Markets Finance and Trade, 41, 53-69.

AZOMAHOU T., NGUYEN VAN P. (2003), "Déforestation, croissance économique et population. Une étude sur données de panel", Revue Economique, 54, 835-855.

AZOMAHOU T., NGUYEN VAN P. (2007), "Nonlinearities and heterogeneity in environmental quality: an empirical analysis of deforestation", Journal of Development Economics, 84, 291-309.

BANQUE MONDIALE (2008), Recueil de statistiques sur les migrations et les envois de fonds, Groupe d'étude des perspectives de développement, Washington DC.

BARBIER E.B. (1994), "The environmental effects of trade in the forestry sector", in OECD, The Environmental Effects of Trade, 55-101.

BARBIER E.B. (2001), "The economics of tropical deforestation and land use: an introduction to the special issue", Land Economics, 77, 155-171.

BARBIER E.B. (2004), "Explaining agricultural land expansion and deforestation in developing countries", American Journal of Agricultural Economics, 86, 1347-1353.

BEINE M., DOCQUIER F., RAPOPORT H. (2008), "Brain drain and human capital formation in developing countries: winners and losers", Economic Journal, 118, 631-652.

BHATTARAI M., HAMMIG M. (2001), "Institutions and the environmental Kuznets curve for deforestation: a cross-country analysis for Latin America, Africa and Asia", World Development, 29, 995-1010.

BHATTARAI M., HAMMIG M. (2004), "Governance, economic policy, and the environmental Kuznets curve for natural tropical forests", Environment and Development Economics, 9, 367-382.

BLACK R. (2001), “Environmental refugees: Myth or reality?”, Working Paper, 34, United Nations High Commissioner for Refugees.

BREUSCH T., PAGAN A. (1980), "The Lagrange multiplier test and its applications to model specification in econometrics", Review of Economic Studies, 47, 239-253.

CHAMI R., FULLENKAMP C., JAHJAH S. (2003), "Are immigrant remittance flows a source of capital for development?", IMF Working Paper, 03/189. 
CHOMITZ K.M., BUYS P., DE LUCA G., THOMAS T.S., WERTZ-KANOUNNIKOFF S. (2007), At Loggerheads? Agricultural expansion, poverty reduction and environment in the tropical forests, World Bank.

COMBES J.L., COMBES MOTEL P., PIRARD R. (2009), “A methodology to estimate impacts of domestic policies on deforestation: Compensated successful efforts for "avoided deforestation (REDD)", Ecological Economics, 68, 680-691.

CONVENTION DE GENEVE (1951), Convention de Genève relative au statut des réfugiés entrée en vigueur le 22 avril 1954, Haut Commissariat des Nations Unies aux droits de l'homme, Genève.

CROPPER M., GRIFFITHS C. (1994), "The interaction of population growth and environmental quality", American Economic Review, 82, 250-254.

CULAS R.J. (2007), "Deforestation and the environmental Kuznets curve: an institutional perspective, Ecological Economics, 61, 429-437.

DEACON R.T. (1994), "Deforestation and the rule of law in a cross-section of countries", Land Economics, 70, 414-430.

DEACON R.T. (1999), "Deforestation and ownership: evidence from historical account and contemporary data", Land Economics, 75, 341-359.

DIDIA D.O. (1997), "Democracy, political instability, and tropical deforestation", Global Environmental Change, 7, 63-76.

DIMOVA R., WOLFF F.C. (2008), "Are private transfers poverty and inequality reducing? Household level evidence from Bulgaria", Journal of Comparative Economics, 36, 584-598.

DOCQUIER F., MARFOUK A. (2006), "International migration by educational attainment (1990-2000)", in Ozden C., Schiff M., eds, International migration, economic development and policy, World Bank and Palgrave Macmillan, New York, 151-200.

EHRHARDT-MARTINEZ K. (1998), "Social determinants of deforestation in developing countries: a cross-national study", Social Forces, 77, 567-586.

EL-HINNAWI E. (1985), Environmental refugees, United Nations Environmental Program, Nairobi.

FAINI R. (2007), "Remittances and the brain drain: Do more skilled migrants remit more?", World Bank Economic Review, 21, 177-191.

FOOD AND AGRICULTURE ORGANIZATION (FAO) (2005), Global Forest Resources Assessment.

GAMMELTOFT P. (2002), "Remittances and other financial flows to developing countries", International Migration, 40, 181-212.

GROSSMAN G.M., KRUEGER A.B. (1995), "Economic growth and the environment", Quarterly Journal of Economics, 60, 353-377.

GUBERT F. (2005), "Migrant remittances and their impact on the economic development of sending countries. The case of Africa", in OCDE, Migrations, remittances and development, Paris, 43-72.

KOOP G., TOLE L. (1999), "Is there an environmental Kuznets curve for deforestation?", Journal of Development Economics, 58, 231-244. 
KOOP G., TOLE L. (2001), “Deforestation, distribution and development”, Global Environmental Change, 11, 193-202.

LACHAUD J.P (1999), "Envois de fonds, inégalité et pauvreté au Burkina Faso", document de travail, Groupe d'Economie du Développement, Université Montesquieu Bordeaux IV.

LIANOS T. (1997), "Factors determining migrant remittances: The case of Greece”, International Migration Review, 31, 72-87.

MCKENZIE D., RAPOPORT H. (2007), "Migration and education inequality in rural Mexico", Integration and Trade Journal, 27, 135-158.

MEYER A., VAN KOOTEN G.C., WANG S. (2003), "Institutional, social and economic roots of deforestation: a cross-country comparison", International Forestry Review, 5, 29-37.

NELSON G.C., HELLERSTEIN D. (1997), "Do roads cause deforestation? Using satellite images in econometric analysis of land use", American Journal of Agricultural Economics, 79, 80-88.

OSILI U.O. (2004), "Migrants and housing investment: Theory and evidence from Nigeria", Economic Development and Cultural Change, 52, 821-850.

RAPOPORT H., DOCQUIER F. (2006), "The economics of migrants' remittances", in Mercier-Ythier J., Kolm S.C., Handbook on the economics of giving, altruism and reciprocity, Elsevier-North Holland, 1135-1198.

RATHA D. (2005), "Workers' remittances: an important and stable source of external development finance", in Mainbo S., Ratha R., Remittances: development impact and future prospects, Washington, World Bank, 19-52.

RUDEL T., ROPER J. (1997), “The paths to rain forest destruction: cross-national patterns of tropical deforestation, 1975-1990", World Development, 25, 53-65.

SELDEN T.M., SONG D. (1994), "Environmental quality and development: is there a Kuznets curve for air pollution emissions?", Journal of Environmental Economics and Management, 27, 147-162.

SHAFIK N. (1994), "Economic development and environmental quality: an econometric analysis", Oxford Economic Papers, 46, 757-773.

STERN N. (2007), The Economics of Climate Change. The Stern Review, Cambridge, Cambridge University Press.

WORLD BANK (2006), At loggerheads? Agricultural expansion, poverty reduction and environment in the tropical forests, Washington DC, World Bank Publications.

YANG D. (2008), "Coping with disaster: The impact of hurricanes on international financial flows, 1970-2002”, B.E. Journal of Economic Analysis and Policy, 8, Article 13. 


\section{ANNEXE 1. LISTE DES PAYS}

\section{Afrique (51 pays)}

Afrique de l'Est et australe : Afrique du Sud, Angola, Botswana, Comores, Kenya, Lesotho, Madagascar, Malawi, Maurice, Mozambique, Namibie, Ouganda, Somalie, Swaziland, Tanzanie, Zambie, Zimbabwe

Afrique du Nord: Algérie, Burkina Faso, Djibouti, Egypte, Erythrée, Ethiopie, Mali, Maroc, Mauritanie, Niger, Soudan, Tchad, Tunisie

Afrique de l'Ouest et centrale : Bénin, Burundi, Cameroun, Cap-Vert, République Centrafricaine, Côte d'Ivoire, République du Congo, République démocratique du Congo, Guinée équatoriale, Gabon, Gambie, Ghana, Guinée, GuinéeBissau, Libéria, Nigéria, Rwanda, Sénégal, Sierra Leone, Togo

\section{Asie et Océanie (27 pays)}

Asie de l'Est : Chine, Mongolie

Asie du Sud et du Sud-Est : Bangladesh, Bhoutan, Cambodge, Inde, Indonésie, Laos, Malaisie, Myanmar, Népal, Pakistan, Philippines, Sri-Lanka, TimorLeste, Thaïlande, Vietnam

Asie de l'Ouest et centrale : Afghanistan, Arménie, Syrie, Tadjikistan, Turquie, Ouzbékistan

Océanie : Fidji, Papouasie-Nouvelle-Guinée, Iles Salomon, Samoa

\section{Amérique et Caraïbes (24 pays)}

Amérique centrale : Costa Rica, Guatemala, Honduras, Mexique, Nicaragua, Panama, Salvador

Amérique du Sud: Argentine, Bolivie, Brésil, Chili, Colombie, Equateur, Paraguay, Pérou, Uruguay, Venezuela

Caraïbes : Cuba, Dominique, Haïti, Jamaïque, Porto Rico, Saint-Vincent-etles-Grenadines, Trinité-et-Tobago 


\section{ANNEXE 2. Top 30 des pays récepteurs de transferts migratoires en 2005}

\begin{tabular}{|c|c|c|c|c|}
\hline & Pays & $\begin{array}{l}\text { Montants transférés } \\
\text { (en millions de dollars) }\end{array}$ & Pays & $\begin{array}{c}\text { Montants } \\
\text { transférés } \\
\text { (en \% du PIB) }\end{array}$ \\
\hline 1 & Mexique & 21657 & Moldavie & 30,79 \\
\hline 2 & Inde & 21293 & Tonga & 30,72 \\
\hline 3 & Chine & 20337 & Guyane & 25,32 \\
\hline 4 & Philippines & 13566 & Lesotho & 22,94 \\
\hline 5 & France & 12306 & Liban & 22,88 \\
\hline 6 & Espagne & 7961 & Haïti & 22,85 \\
\hline 7 & Belgique & 7241 & Honduras & 21,64 \\
\hline 8 & Allemagne & 6363 & Tadjikistan & 20,16 \\
\hline 9 & Royaume-Uni & 6302 & Jordanie & 19,82 \\
\hline 10 & Indonésie & 5419 & Arménie & 19,18 \\
\hline 11 & Egypte & 5017 & Bosnie-Herzégovine & 19,06 \\
\hline 12 & Liban & 4924 & Jamaïque & 18,35 \\
\hline 13 & Roumanie & 4732 & Serbie-Monténégro & 17,73 \\
\hline 14 & Serbie-Monténégro & 4650 & Salvador & 16,65 \\
\hline 15 & Maroc & 4589 & Albanie & 15,40 \\
\hline 16 & Bangladesh & 4314 & Territoires palestiniens & 14,90 \\
\hline 17 & Pakistan & 4280 & Népal & 14,80 \\
\hline 18 & Vietnam & 4000 & Philippines & 13,74 \\
\hline 19 & Pologne & 3549 & Cap-Vert & 13,71 \\
\hline 20 & Brésil & 3540 & Kirghizistan & 13,09 \\
\hline 21 & Colombie & 3345 & Gambie & 12,44 \\
\hline 22 & Nigeria & 3329 & Nicaragua & 12,36 \\
\hline 23 & Portugal & 3101 & Kiribati & 10,55 \\
\hline 24 & Guatemala & 3033 & Guatemala & 9,56 \\
\hline 25 & Australie & 2989 & Guinée-Bissau & 9,30 \\
\hline 26 & Autriche & 2941 & République dominicaine & 9,22 \\
\hline 27 & Russie & 2918 & Togo & 9,13 \\
\hline 28 & États-Unis & 2890 & Sri Lanka & 8,46 \\
\hline 29 & Salvador & 2842 & Mongolie & 7,82 \\
\hline 30 & République dominicaine & 2719 & Maroc & 7,78 \\
\hline
\end{tabular}

Source : Recueil de statistiques sur les migrations et les envois de fonds, Banque Mondiale, 2008.

Note: Les transferts migratoires sont exprimés à prix courants. 